\title{
ANÁLISE DE APLICATIVOS PARA COMUNICAÇÃO AUMENTATIVA EALTERNATIVA A INDIVÍDUOS COM TRANSTORNO DO ESPECTRO AUTISTA
}

\author{
ANALYSIS OF APPLICATIONS FOR EVERYTHING EFFECTIVE \\ COMMUNICATION FOR INDIVIDUALS WITH AUTISTIC SPECTRUM \\ DISORDERS
}

Fábio Madeira Peres ${ }^{1}$

Danúbia Espíndola², D.Sc

Regina Bärwaldt ${ }^{3}$, D.Sc

Raquel Machado ${ }^{4}$

Thiago Grilo ${ }^{5}$

(1) Universidade Federal do Rio Grande (FURG) e-mail:fabiomadeira@furg.br

(2) Universidade Federal do Rio Grande (FURG) e-mail: danubiaespindola@furg.br

(3) Universidade Federal do Rio Grande (FURG) e-mail: reginabarwaldt@furg.br

(4) Universidade Federal do Rio Grande (FURG) e-mail: tpraquel@gmail.com

(5) Universidade Federal do Rio Grande (FURG) e-mail: thiagopiresgrilo@hotmail.com

Palavras-chave: Comunicação Aumentativa e Alternativa, Tecnologias Assistivas, Interface Humano Computador 


\section{$16^{\circ}$ \\ ERGODESIGN USIHC CINAHPA}

$16^{\circ}$ Ergodesign - Congresso Internacional de Ergonomia e Usabilidade de Interfaces Humano Tecnológica: Produto, Informações Ambientes Construídos e Transporte

$16^{\circ}$ USIHC - Congresso Internacional de Ergonomia e Usabilidade de Interfaces Humano Computador

CINAHPA | 2017 - Congresso Internacional de Ambientes Hipermídia para Aprendizagem.

Resumo: Este artigo resulta da avaliação de interface de quatro aplicativos de comunicação aumentativa e alternativa (CAA), direcionadas para pessoas com transtorno do espectro autista (TEA). Para tanto, foram pesquisadas interfaces e aplicativos direcionados ao público alvo em questão, para serem utilizadas em espaços educativos formais, não formais e outros. A metodologia parte da adaptação de três questionários: Ergolist, SUMI e EduCatalog. A análise foi realizada por meio de um Checklist, submetido ao julgamento de cinco especialistas em tecnologia da informação. Evidenciaram-se, através da análise das questões, alguns quesitos importantes a serem aperfeiçoados, bem como qualidades desejáveis para a utilização proveitosa das ferramentas.

\section{Key-words: Augmentative and Alternative Communication, Assistive Technologies, Human Computer Interface}

This article results from the assessment of four augmentative communication applications and alternative interface $(C A A)$, directed to people with autism spectrum disorder (ASD). Therefore, interfaces and applications targeted to the target audience in question were surveyed, for usein formal educational settings, formal and others. The methodology of the adaptation of three questionnaires: Ergolist, SUMI and EduCatalog. The analysis was performed by means of a checklist, submitted to the judgment of five experts in information technology. They showed up, through the analysis of the issues, some important questions to be improved and desirable qualities for the profitable use of tools.

\section{Introdução}

Conforme a National Autistic Society (NAS), principal instituição do Reino Unido no auxílio a pessoas com Transtorno do Espectro Autista (TEA) e suas famílias, o autismo é um transtorno no desenvolvimento que impacta a maneira como a pessoa se comunica e se relaciona socialmente. KIRST faz uma ressalva importante a respeito da comunicação, associando ao universo do TEA:

"É importante lembrar que a comunicação e a interação não envolvem necessariamente o uso de linguagem falada. Muitas crianças com TEA apresentam atrasos nessas áreas e enquanto não souberem utilizar a linguagem falada, outros métodos de comunicação precisam ser estabelecidos". (2015, p. 27). As pessoas com TEA apresentam dificuldades na linguagem verbal e não verbal, sendo que quando estão se comunicando, geralmente consideram que as pessoas querem dizer exatamente aquilo que dizem, ou seja, compreendem a linguagem de uma maneira extremamente literal. BONOTTO \& KIRST relatam que os indivíduos com TEA "Geralmente entendem o que as outras pessoas lhe dizem, mas elas próprias utilizam meios alternativos de comunicação, como linguagem de sinais ou símbolos visuais" (2014, p. 8).

$\mathrm{Na}$ busca pelo rompimento de barreiras impostas a indivíduos que sofrem danos causados por distúrbios físicos e/ou cognitivos, o homem desenvolveu algumas ferramentas capazes de suprir as necessidades desencadeadas por esses distúrbios. A esses instrumentos de apoio, denominamos Tecnologias Assistivas (TA). Existe uma área da TA, denominada de Comunicação Aumentativa e Alternativa (CAA), definida por BERSCH \& SCHIRMER, como uma área "destinada a pessoas sem fala ou sem escrita funcional ou em defasagem entre sua necessidade comunicativa e sua habilidade em falar e/ou escrever" (2005, p. 6). A CAA busca uma valorização de todas as maneiras de expressões dos indivíduos com dificuldades de comunicação, pois estas formas são maximizadas e possibilitam a criação de novos canais de comunicação. "A criança autista é um exemplo prototípico de um indivíduo com inteligência intrapessoal prejudicada; na verdade, essas crianças talvez nunca tenham sido capazes de se referirem a si mesmas. Ao mesmo tempo, elas frequentemente apresentam notáveis capacidades dos domínios musical, computacional, espacial ou mecânico" (GARDNER, 1995, p.29).

A capacidade de domínio computacional, citado pelo autor acima, pode ser um aliado no desenvolvimento da comunicação entre portadores
Realização:

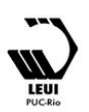

UNIVERSIDADE FEDERAL DE SANTA CATARINA 


\section{$16^{\circ}$ \\ ERGODESIGN USIHC CINAHPA}

do TEA e educadores em espaços voltados para a inclusão. O computador como ferramenta interativa, através de seus inumeráveis recursos, pode respaldar e proporcionar uma relação conversacional de forma mais objetiva e concreta. Para tanto, a interação humano-computador entra em ação, ciência esta que estuda a relação entre pessoas e interfaces computacionais, de forma interdisciplinar, envolvendo, além da computação, áreas como a semiótica, psicologia, ergonomia, artes, design, linguística, sociologia, entre outras.

Bergeson (2003) recomenda para a interação com sujeitos autistas, principalmente aqueles que não se comunicam verbalmente, ferramentas que favoreçam o diálogo por meios alternativos e suplementares, enfatizando a importância do aproveitamento dos interesses do indivíduo. A interface deve proporcionar tranquilidade, através de ícones que possam ser reconhecidos, oportunizando a interlocução de forma espontânea e intuitiva.

O presente artigo tem como objetivo avaliar quatro aplicativos mobile destinados a CAA com o intuito de destacar as qualidades e os defeitos de cada um durante a interação, vislumbrando elencar conjuntos de características que seriam desejáveis ou não num aplicativo resultante de todos os avaliados. Um Checklist foi submetido a cinco especialistas em Tecnologias da Informação (TI), dois com formação pedagógica, sendo os resultados discutidos e apresentados de forma discursiva. Por fim, os aplicativos selecionados para a análise, o Checklist adaptado e os resultados e discussões da proposta são apresentados a seguir.

\subsection{Aplicativos selecionados para avaliação}

A pesquisa contemplou ferramentas de autoria e freeware, disponíveis nas plataformas Android e IOS destinados à CAA. Quatro dos aplicativos utilizados na avaliação foram eleitos por uma equipe de especialistas em TEA ${ }^{1}$, sendo estes que mais se aproximam em termos de interface e funcionalidades desejáveis para um utilitário destinado ao público com TEA.

\footnotetext{
${ }^{1}$ Esta equipe pertence a uma escola especializada em autismo.
}

$16^{\circ}$ Ergodesign - Congresso Internacional de Ergonomia e Usabilidade de Interfaces Humano Tecnológica: Produto, Informações Ambientes Construídos e Transporte

$16^{\circ}$ USIHC - Congresso Internacional de Ergonomia e Usabilidade de Interfaces Humano Computador

CINAHPA | 2017 - Congresso Internacional de Ambientes Hipermídia para Aprendizagem.

SCALA $^{2}$ : fornece ao usuário a construção de pranchas de comunicação, com ou sem ajuda de terceiros, expressa emoções, necessidades, indica objetos ou adiciona imagens próprias. Dispõe de 5000 figuras ilustrativas em seu banco de dados.

LetMe Talk ${ }^{3}$ : possui 9000 imagens oriundas do sistema pictográfico de livre distribuição ARASAAC $^{4}$, apresenta a opção de incluir e capturar imagens do dispositivo.

Vox4All ${ }^{5}$ : com mais de 11 mil símbolos pictográficos, este aplicativo é baseado em pranchas de comunicação, sendo que cada uma das células das pranchas pode exibir símbolos, textos, fotos capturadas, gravações de voz, texto para leitura, e, também, englobar outras pranchas.

PictoDroid Lite ${ }^{6}$ : contendo imagens oriundas do ARASAAC, contempla expressões específicas, como por exemplo: "eu quero beber..., eu quero comer..." e verbalização das imagens selecionadas.

\section{Metodologia}

A metodologia utilizada resultou num Checklist adaptado de três outras avaliações, contemplando questões específicas no processo avaliativo. Tais julgamentos foram escolhidos por abordarem questões pontuais sobre interfaces e suas funcionalidades, facilitando a identificação de carências e apontando com clareza pontos que devam ser aprimorados. As adaptações foram necessárias, já que algumas questões foram irrelevantes para o processo avaliativo. Os questionários são os seguintes:

$\mathrm{SUMI}^{7}$ : composto por cinquenta questões. Pode ser adaptado de acordo com as necessidades de cada desenvolvedor/avaliador.

\footnotetext{
${ }^{2}$ Maiores informações: 〈http://scala.ufrgs.br>

${ }^{3}$ Maiores informações:

<http://www.letmetalk.info/pt.html>

${ }^{4}$ Maiores informações: <http://arasaac.org>

${ }^{5}$ Maiores informações: 〈http://vox4all.imagina.pt>

${ }^{6}$ Maiores informações:

<http://www.accegal.org/pictodroid-lite>

${ }^{7}$ Maiores informações:

<http://www.ucc.ie/hfrg/questionnaires/sumi/index.html
} 


\section{$16^{\circ}$ \\ ERGODESIGN USIHC CINAHPA}

$16^{\circ}$ Ergodesign - Congresso Internacional de Ergonomia e Usabilidade de Interfaces Humano Tecnológica: Produto, Informações Ambientes Construídos e Transporte

$16^{\circ}$ USIHC - Congresso Internacional de Ergonomia e Usabilidade de Interfaces Humano Computador

CINAHPA | 2017 - Congresso Internacional de Ambientes Hipermídia para Aprendizagem.
EduCatalog: composto por uma lista de requisitos direcionados para o levantamento de dados e desenvolvimento de ferramentas educacionais interativas. Envolve três itens essenciais para a construção da proposta: Teoria da Aprendizagem, Requisitos Pedagógicos e Descrição dos Requisitos (HENRIQUE, 2016).

Ergolist $^{8}$ : apresenta módulos que se dividem em: justificativas, exemplos de recomendações, comentários, Checklists, questões e glossário.

O Checklist proposto para esta análise contém dezenove questões objetivas, com opções de resposta (sim ou não), além de uma discursiva. Tais questões foram encapsuladas num formulário eletrônico ${ }^{9}$, replicado um para cada aplicativo, enviadas para cinco avaliadores ${ }^{10}$ especialistas.

O número de avaliadores convidados para responder foi baseado nas recomendações de Nielsen (1994), evidenciando empiricamente que " 05 avaliadores identificam cerca de $75 \%$ dos problemas totais de usabilidade na interface".

Salienta-se ainda, que neste processo de aplicação não foram convidados indivíduos com TEA intencionando preservar o bem estar dos mesmos, impedindo de ocasionar os sintomas gerais de estresse.

\section{Resultados e Discussão}

Os resultados mais expressivos do Checklist são apresentados a seguir.

1) $\mathrm{O}$ app oferece um feedback imediato e adequado a cada ação do usuário?

Os aplicativos SCALA e LetMe Talk apresentaram baixo feedback imediato e adequado a cada ação do usuário. Segundo as 10 Heurísticas de Nielsen

\footnotetext{
${ }^{8}$ Maiores informações:

<http://labiutil.inf.ufsc.br/ergolist/check.html>

${ }^{9}$ Os formulários podem ser consultados em: SCALA:

<http://goo.gl/forms/6WqgtQDQSw>;

LetMe Talk:

<http://goo.gl/forms/oBJjp7vC9F3yhNzS2>; Vox4All: <http://goo.gl/forms/EVU2HC6tGF>; PictoDroid Lite: $<$ http://goo.gl/forms/HCexCvJH3f >

${ }^{10}$ Os perfis dos avaliadores: um Doutor em Informática na Educação, três Mestrandos em Engenharia de Computação e um Graduando em Sistemas de Informação.
}

(1994), feedbacks imediatos, fornecidos num tempo razoável, tornam os usuários mais informados sobre o que está acontecendo e consequentemente mantém maior visibilidade sobre o status do sistema.

2) Os componentes interativos selecionados são claramente distintos dos demais?

$\mathrm{O}$ aplicativo SCALA obteve o melhor desempenho, apresentando $80 \%$ de aprovação. Em contrapartida, o aplicativo que ficou com maior índice de rejeição foi o Vox4All, com $80 \%$ dos avaliadores respondendo não para esta questão. A ausência da clareza de seleção dos componentes interativos pode comprometer o contexto da informação apresentada.

3) $\mathrm{O}$ app funciona corretamente, sem apresentar problemas durante as interações?

O resultado obtido nesta questão foi significativo para o aplicativo SCALA. Todos os avaliadores relataram que o mesmo não funcionou corretamente, apresentando problemas quando o usuário interagia. Foi constatado que ao instalar, em dispositivos móveis menores do que 8 ", as seleções das categorias ficavam parcialmente visíveis, impedindo o funcionamento correto.

4) O significado de símbolos, ícones e imagens são compreensíveis e intuitivos?

Dentre os aplicativos avaliados, o LetMe Talk apresentou o pior desempenho, uma vez que os símbolos e significados não transmitem interpretações claras em alguns momentos. Conforme CYBIS (2007) relata sobre os princípios ergonômicos para interfaces humano-computador, quando os códigos e denominações não estão em conformidade, existem chances de levar os usuários a cometerem erros, como por exemplo, escolherem opções erradas ou deixar de informar um dado importante. Ainda segundo o autor, quando a codificação e as denominações são significativas, a recordação e o reconhecimento são mais fáceis de serem assimilados pelos usuários. 5) As fontes, textos, símbolos e imagens apresentam contraste suficiente em relação ao plano de fundo e detalhamento favoráveis à leitura em tela pequena?

Todos os aplicativos obtiveram bons resultados nesta questão, ficando todos acima dos $60 \%$, indicando que todos aplicativos colocaram em prática os conceitos envolvidos em tal questão. 


\section{$16^{\circ}$ \\ ERGODESIGN USIHC CINAHPA}

CYBIS (2007) relata, sobre as telas pequenas nos ambientes mobile, que "... pode tornar a leitura mais difícil, exigindo novos modos de apresentação do conteúdo para a maximização de espaço sem sobrecarregar a área de visualização" (p. 227).

6) É possível retornar a tela anterior a qualquer momento?

$\mathrm{O}$ aplicativo LetMe Talk obteve unanimidade positiva dos avaliadores. Em contrapartida, PictoDroid Lite atingiu o pior desempenho nesta questão, justamente pelo fato do aplicativo não apresentar tal funcionalidade. Conforme as Heurísticas de NIELSEN (1994) no que se refere ao controle do usuário e liberdade, os usuários precisam ter um modo fácil para sair de um estado indesejado, utilizado por engano ou não, sem ter que percorrer longos caminhos no sistema. CYBIS (2007) faz algumas recomendações direcionadas para a interação em ambientes mobile, dentre elas a facilidade da navegação. $\mathrm{O}$ autor relata que "a função Voltar à tela anterior é muito importante para o usuário móvel, devendo estar sempre acessível e visível" (p. 237).

7) A customização do app é evidente para o usuário?

Os resultados foram negativos para todos os aplicativos avaliados, uma vez que em nenhum ficou evidente a sua customização. Isto não significa que as ferramentas não possuem tal funcionalidade, mas sim que estas não estão facilitadas para os usuários.

8) $\mathrm{O}$ app possui navegação intuitiva a tal ponto que as tarefas são relativamente simples de serem executadas, deixando claro quais são os próximos passos para realizar a tarefa? O aplicativo LetMe Talk obteve unanimidade positiva dos avaliadores. Em contrapartida, PictoDroid Lite atingiu o pior desempenho, justamente pelo fato do aplicativo não apresentar tal funcionalidade. Conforme as Heurísticas de Nielsen (1994) no que se refere ao controle do usuário e liberdade, os usuários precisam ter um modo fácil para sair de um estado indesejado, utilizado por engano ou não, sem ter que percorrer longos caminhos no sistema. CYBIS (2007) faz algumas recomendações direcionadas para a interação em ambientes mobile, dentre elas a facilidade da navegação. $\mathrm{O}$ autor relata que "a função voltar à tela anterior é muito importante $16^{\circ}$ Ergodesign - Congresso Internacional de Ergonomia e Usabilidade de Interfaces Humano Tecnológica: Produto, Informações Ambientes Construídos e Transporte

$16^{\circ}$ USIHC - Congresso Internacional de Ergonomia e Usabilidade de Interfaces Humano Computador

CINAHPA | 2017 - Congresso Internacional de Ambientes Hipermídia para Aprendizagem.

para o usuário móvel, devendo estar sempre acessível e visível" (p. 237).

9) É possível controlar a sequência de ações realizadas?

Uma das perguntas com maior rejeição, a preponderância das ferramentas não oferece opção de cancelamento para uma atividade que está sendo representada. O Vox4All e o PictoDroid Lite obtiveram $100 \%$ de desaprovação, enquanto que o SCALA e LetMe Talk não ultrapassaram a marca dos $20 \%$ de positividade, pois a partir do momento que uma ação é inicializada, não há opção de revogação, levando ao indivíduo que está usufruindo da ferramenta ter que aguardar a finalização da operação para poder dar prosseguimento.

10) A experiência de uso é considerada pelo app? O SCALA obteve a pior avaliação neste item, recebendo $0 \%$ de parecer favorável. Logo a seguir encontram-se LetMe Talk, Vox4All e PictoDroid Lite na mesma posição, com apenas $20 \%$ de ponderação positiva. Por se tratar de aplicativos direcionados a pessoas com dificuldades cognitivas, de interação e assimilação, este item não foi levado em conta na elaboração da ferramenta. O Labiuti $1^{11}$ reforça esta questão, manifestando-se dentro dos critérios ergonômicos, considerando a experiência do usuário: "Pode-se fornecer aos usuários inexperientes diálogos fortemente conduzidos, ou mesmo passo a passo. 11) O app possui ajuda ou documentação? O SCALA foi o que conquistou um julgamento mais assertivo, onde $80 \%$ dos especialistas opinaram concordantemente. Há um menu "ajuda" com tutorial disponível através de módulos, com orientações para configuração e criação de histórias personalizadas. Vox4All e LetMe Talk atingiram $40 \%$ de assertividade e o PictoDroid Lite adquiriu a rejeição de $80 \%$ do julgamento especialista. Por não ser um instrumento considerado "comum", um documento com ajuda é imprescindível para que a ferramenta seja comumente manuseada para o seu devido fim. 12) Você recomendaria este app para pessoas com transtorno do espectro autista?

O mais recomendável, segundo os especialistas, é

\footnotetext{
${ }^{11}$ Maiores informações:

$<$ http://www.labiutil.inf.ufsc.br>
}

Realização:

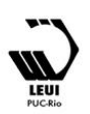




\section{$16^{\circ}$ \\ ERGODESIGN USIHC CINAHPA}

o LetMe Talk com $60 \%$ de análise concordante. SCALA e PictoDroid Lite somente $20 \%$ indicariam como sugestão, enquanto que o Vox4All, com avaliação $100 \%$ negativa, não seria proposto por nenhum dos avaliadores.

\section{Conclusão}

Conforme pode ser observado com os resultados obtidos no Checklist adaptado, existiram diversas questões que obtiveram baixas avaliações por não serem tratadas da melhor maneira nos aplicativos elencados. Pelo fato dos indivíduos com TEA possuírem comportamentos repetitivos e restritivos, existe uma grande dificuldade em sugerir mudanças nos seus hábitos e consequentemente na idealização de ferramentas funcionais específicas direcionadas para esse público. Com a avaliação concluída foi possível perceber que alguns quesitos podem ser aprimorados, tanto na interface, quanto na eficiência, como por exemplo: feedback imediato, sequências das ações realizadas, customização, exposição de informações realmente indispensáveis, apresentação de funções mais utilizadas, experiência do usuário, ajuda ou documentação e, principalmente, as ações recomendadas.

Porém, outros quesitos foram bem avaliados e contemplados de forma satisfatória: a clareza das seleções dos componentes interativos, o significado dos símbolos, ícones e imagens, o contraste das fontes, textos, símbolos e imagens em relação ao plano de fundo, a retomada a tela anterior, a navegação intuitiva e a responsividade. Por fim, com a aplicação e análise do Checklist adaptado, foi possível elencar características desejáveis para um aplicativo resultante dos avaliados. Através do auxílio de artefatos tecnológicos, podemos melhorar as relações sociais de indivíduos com TEA em espaços educacionais formais, não formais e outros, e que, apesar dos obstáculos que este público enfrenta, podem conquistar autonomia, uma vez que os incentivos colaboram para o seu aperfeiçoamento como ser humano, fazendo com que o indivíduo se sinta capaz de explorar o mundo ao qual pertence. $16^{\circ}$ Ergodesign - Congresso Internacional de Ergonomia e Usabilidade de Interfaces Humano Tecnológica: Produto, Informações Ambientes Construídos e Transporte

$16^{\circ}$ USIHC - Congresso Internacional de Ergonomia e Usabilidade de Interfaces Humano Computador

CINAHPA | 2017 - Congresso Internacional de Ambientes Hipermídia para Aprendizagem.

\section{BIBLIOGRAFIA}

Bergeson, T. Los aspectos pedagógicos de lós transtornos del espectro autista. Espanha: [s.n.], 2003.

\section{BERSCH, R \& SCHIRMER, C. Tecnologia} Assistiva no processo educacional. In: BRASIL. Ministério da Educação. Ensaios Pedagógicos: construindo escolas inclusivas. Brasília: MEC/SEESP, 2005.

BONOTTO, R. C. \& KIRST, N. O que é autismo e como reconhecê-lo. Volume 6. Cadernos Pandorga de Autismo - Volume 6. São Leopoldo: Oikos, 2014.

CYBIS, W. Ergonomia e usabilidade: conhecimento, métodos e aplicação. Walter Cybis, Adriana Holtz Betiol, Richard Faust. São Paulo: Novatec Editora, 2007.

GARDNER, H. Inteligências Múltiplas: a teoria na prática. Trad. Maria Adriana Veríssimo Veronese. Porto Alegre: Artes Médicas, 1995.

HENRIQUE, S. M. EduCatalog 4re: um catálogo de requisitos para auxiliar o desenvolvimento de softwares educacionais. 2016. Dissertação (Mestrado em Ciência da Computação) Universidade Federal de Pernambuco, Recife. 2016.

\section{LABIUTIL. Critérios Ergonômicos. Disponível em}

<http://www.labiutil.inf.ufsc.br/CriteriosErgonomi cos/LabIUtil2003-Crit/420adaptb_A.html>. Acesso em: 14 de Mar de 2017.

KIRST, N. Autismo: organização, sequenciamento e priorização; comportamentos complicados; comunicação e interação.

Cadernos Pandorga de Autismo - Volume 8. São

Leopoldo: Oikos, 2015.

NIELSEN, J. Usability engineering. San

Francisco: Morgan Kaufman. 1994.

Realização:

